\title{
USER-CENTERED DESIGN FOR GLOBAL INFORMATION SYSTEMS
}

\author{
Susan E. Conners, Ph.D, Purdue University Calumet, conners@calumet.purdue.edu \\ Roy O. Foreman, Purdue University Calumet, foreman@calumet.purdue.edu
}

\begin{abstract}
User-centered design is rapidly becoming a necessary component of business systems. As users become more sophisticated, they expect usability to be a key component of information systems. Global information systems have more challenges to create systems with user-centered design principles as they must account for different languages and cultures. This study identifies key user-centered design principles and then measures the websites of twenty Fortune 500 companies with an international presence against these principles.
\end{abstract}

Keywords: User-centered design, global information systems, usability, information systems

\section{INTRODUCTION}

Global information systems can be defined as electronic systems accessible on an international scale and reaching users at different locations and from different cultures and languages. Corporate web sites serve this purpose. Large international companies use their websites to communicate with the customers and constituents around the world. The importance of providing users with websites that are effective and meet user's requirements is crucial. [1] This paper evaluates a cross section of corporate web sites against usability criteria.

The review of literature on this topic indicates a small amount of research is available and many of the articles are published in trade journals rather than traditional academic journals. Due to these limitations the references are primarily white papers and trade journals. Information on the criteria is more readily available than specific information on the global aspects of this topic.

\section{Web Sites and Usability}

Corporations have discovered that user-centered design is critical to successful software design. Benefits of user centered design include increased sales, reduced support costs, avoiding unnecessary functions, and expediting decision making. [1] Evidence of this growing trend can be found in the practice of World Usability Day. This event received media coverage on five continents and in thirty five countries. [5] Corporate web sites are a highly visible type of software representing companies to the general public. These websites are arguably the most important place to implement user-centered design principles. To be successful, each website requires a careful business analysis before designing the interface. [7] Many companies conduct surveys and focus groups and even use high-technology eyetracking devices to uncover how customers use a Web site and how their experiences affect feelings about the parent company. [3] This was the premise used to evaluate the criteria for the website evaluation. The web sites examined in this research are compared against basic usability criteria.

\section{RESEARCH METHODOLOGY}

This research focused on the following research question: Do corporate websites of Fortune 500 international companies meet the basic standards for user-centered design?

The research hypotheses are as follows:

$\mathrm{H}_{1}$ : Corporate websites of Fortune 500 companies doing business internationally meet basic usability criteria.

$\mathrm{H}_{2}$ : Websites of international companies demonstrate an awareness of the needs of international users.

There were three main components to the structure of the research: the companies selected, the criteria used and the rating scale.

\section{Selection Criteria}

The companies chosen were Fortune 500 international companies. A cross section of industries is represented including automotive, financial, software, manufacturing, airline, oil, and shipping companies. These twenty companies are well known around the world and do business in multiple countries.

The user base for these companies includes several nationalities, both genders and various age groups. Since these websites represent the company to the general public, companies were chosen with a wide user base for evaluation. 
The selection of criteria for the research is based on documented usability criteria. Usability focuses on the interface, elements, and quality of ease of use. [7] The literature reviewed revealed many factors of usability. There were several criteria that stood out in the review. A Journal of Electronic Commerce article identifies the top three reasons for users to abandon a website as: (1) could not find item or (2) information, site is disorganized, and (3) pages downloaded too slowly. [6] These items were incorporated into the research criteria. Additional studies further refined these broader categories to navigation capability, search availability within the site, layouts that are consistent and easy to use. [4]

The international audience for these sites required that criteria to judge the websites include this aspect of the websites. The design of websites is not a one size fits all when you are working with an international audience. [2] Cultural aspects must be taken into consideration. In human computer interfaces, culture is defined as common values, attitudes and behavioral pattern shared by people and influencing how they interact with computers. [8] Criteria evaluating the ability to access multiple languages and the use of internationally recognized images and symbols were added to the evaluation to address this issue. The following table listed the criteria and definitions:

Table1 Evaluation Criteria

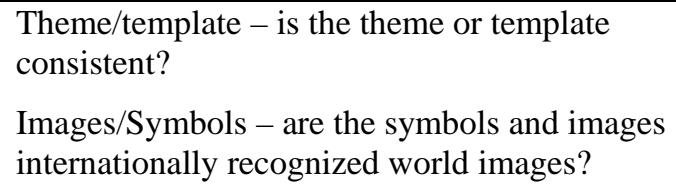

Language - are there multiple languages available to access the site?

Load time - no longer than 10 seconds on high speed line?

Instructions - short instructions that require less than 30 seconds to read?

Navigation memory - does the site remember the last page or instruction?

Search capability - does the site allow searches from home page?

Grouping - are like items grouped together and groups differentiated by color, size, or location? Organization - is there logical progression through the site?

The websites were evaluated by the authors using a rating scale of 1 to 3 with three being good, two indicating average and one being poor using the criteria outlined above. The criteria address both research questions and provide indicators of areas of success and shortcomings.

After viewing and rating all twenty websites, a matrix was constructed with the ratings. The matrix includes the rating each company received on each of the criteria. The results are illustrated in the Figure 1 matrix.

\section{RESULTS}

The data overall indicated a favorable result for the companies. The results were reviewed and indicted some widespread strength, and some weaknesses. In Table 2 below, the averages of the criteria across all companies are listed. These results depict the comprehensive averages.

Table 2 Criteria Averages

\begin{tabular}{lc} 
Criteria & Average \\
\hline Theme/template & 2.5 \\
Images/Symbols & 2.1 \\
Language & 1.9 \\
Load time & 3.0 \\
Instructions & 2.6 \\
Navigation & 2.7 \\
Search & 2.6 \\
Grouping & 2.3 \\
Organization & 2.4
\end{tabular}

The data shows that all sites met the technical requirement for load time and the higher scores were in technical areas such as navigation and search capabilities. This seems to indicate the role of the IT technicians in corporate web site development. The lower scores were in language and internationally recognized images and symbols. These lower scoring items are usability issues rather than technical issues.

The scores for individual companies ranged from a low of 2.0 to a high of 3.0. There were three companies that had 3.0 ratings in all criteria areas. These three perfect scores came from different sectors of industry.

The companies with lower average scores all had failures in similar areas. The low scores were primarily in language, images, and symbols. 
Figure 1. Comparison Matrix

\begin{tabular}{|c|c|c|c|c|c|c|c|c|c|c|c|c|c|c|c|c|c|c|c|c|}
\hline Companies & $\# 1$ & \#2 & \#3 & \#4 & \#5 & \#6 & \#7 & \#8 & $\# 9$ & \#10 & \#11 & $\# 12$ & $\# 13$ & \#14 & \#15 & \#16 & \#17 & \#18 & \#19 & \#20 \\
\hline \multicolumn{21}{|l|}{ Criteria } \\
\hline \multicolumn{21}{|l|}{ Site Design } \\
\hline theme/template & 3 & 2 & 2 & 3 & 3 & 3 & 1 & 1 & 3 & 2 & 2 & 2 & 3 & 3 & 2 & 3 & 3 & 3 & 3 & 3 \\
\hline images/symbols & 3 & 3 & 3 & 3 & 3 & 3 & 3 & 2 & 3 & 2 & 2 & 1 & 2 & 1 & 1 & 1 & 2 & 1 & 1 & 1 \\
\hline language & 3 & 3 & 2 & 1 & 3 & 3 & 3 & 3 & 3 & 1 & 2 & 1 & 1 & 1 & 2 & 1 & 1 & 1 & 1 & 1 \\
\hline \multicolumn{21}{|l|}{ Navigation } \\
\hline load time & 3 & 2 & 3 & 3 & 3 & 3 & 3 & 3 & 3 & 3 & 3 & 3 & 3 & 3 & 3 & 3 & 3 & 3 & 3 & 3 \\
\hline instructions & 3 & 3 & 3 & 3 & 3 & 3 & 3 & 3 & 3 & 2 & 2 & 2 & 2 & 3 & 2 & 2 & 2 & 2 & 2 & 3 \\
\hline navigation memory & 3 & 3 & 3 & 3 & 3 & 3 & 3 & 3 & 3 & 3 & 2 & 2 & 2 & 3 & 2 & 3 & 2 & 2 & 2 & 3 \\
\hline \multicolumn{21}{|l|}{ Locate Information } \\
\hline search capability & 3 & 3 & 1 & 3 & 3 & 3 & 3 & 1 & 2 & 2 & 2 & 3 & 1 & 3 & 3 & 3 & 3 & 3 & 3 & 3 \\
\hline grouping & 3 & 3 & 2 & 2 & 3 & 3 & 3 & 2 & 2 & 2 & 3 & 2 & 2 & 2 & 2 & 2 & 2 & 2 & 2 & 2 \\
\hline organization & 3 & 3 & 2 & 2 & 3 & 3 & 2 & 2 & 2 & 2 & 2 & 2 & 3 & 3 & 2 & 3 & 2 & 2 & 2 & 3 \\
\hline
\end{tabular}

\section{CONCLUSIONS}

This research was not comprehensive but was intended to determine if there were problems in usability design across international corporate websites. It was clear from the ratings that failure to recognize the international audience was a shortcoming in several instances. The indicators were the poor scores in language and international symbols. While the websites overall met basic user centered design criteria, there were indications of problems designing for the broader audience or not recognizing the broader audience for the website.

Corporations need to investigate the actual audience and their usability requirements when designing websites. The usability staff they employ must recognize that these websites are the global face of the company. In the future, more extensive research on a larger number of corporate websites needs to be undertaken.

Based upon the results of our study, we concluded that corporate web sites of sample international Fortune 500 companies rated satisfactorily on the user centered design criteria. This is significant because it supports the industry trend to hire usability experts and employ the user centered design techniques in their software development plan for global systems.

\section{REFERENCES}

1. Agrawal, H., Thosar, A.(2004). Institutionalizing usability at Anthem. White Paper Human Factors International. Available: www.humanfactors.com

2. Apala, L. (2005). Another culture, another method. Proceedings of the HCII2005 Conference, Mahwah, NJ USA.

3. Bannan, K. (2002). Helping business evaluate their Internet presence. The New York Times

4. Malecek, P. (2004). From inspiration to action at A.G. Edwards \& Sons, Inc., the prescription and practice for institutionalizing usability. White Paper Human Factors International. Available: www.humanfactors.com.

5. Rosenzweig, E. (2006). World usability day: a challenge for everyone. Journal of Usability Studies. Vol 1 Issue 4

6. Schaffer, E., Sorflaten, J. (1999). Web usability illustrated: breathing easier with you usable ecommerce site. Journal of Electronic Commerce. Vol. 11 N. 4.

7. Singh, S. (2003) Keep out the clutter, Businessworld

8. Vatrapu, R., Perez-Quinones, M. (2006). Culture and usability evaluation: the effects of culture in structured interviews. Journal of Usability Studies. Vol 1 Issue 4

9. Weinschenk, S. (2005). Usability a business case. White Paper Human Factors International. Available: www.humanfactors.com 